\title{
ON THE CONVERGENCE OF APPROXIMATE SOLUTIONS OF THE HEAT EQUATION TO THE EXACT SOLUTION
}

\author{
WERNER LEUTERT
}

Introduction. In many cases it is practically impossible to solve an initial value problem for a partial differential equation exactly although it can be proved that the exact solution does exist and is uniquely determined.

Therefore the partial differential equation is very of ten replaced by a difference equation which is easier to solve and which furnishes an approximation to the solution of the original problem. Three questions arise immediately.

(a) If $x$ and $t$ are the independent variables, does the mesh ratio $r$ $\left(r=\Delta t /(\Delta x)^{2}\right.$ in the parabolic case) have any influence on the convergence or stability of the approximate solution?

(b) Which one of the difference equations leading to the differential equation will furnish a good approximation?

(c) How do the initial values of the problem for the difference equation have to be chosen in order to furnish a good approximation?

The author believes that the first question has been overemphasized while the importance of the second and third has not been fully realized. This is primarily due to the fact that, in the paper by O'Brien, Hyman, and Kaplan [1 $]^{1}$ where von Neumann's test of stability is introduced into the literature, it is erroneously stated that a positive answer to von Neumann's test is necessary and sufficient for convergence. As an example it is pointed out in their paper that the numerical solution of Richardson [2] for the problem of finding the temperature in a slab with faces at temperature zero does not converge to the exact solution because von Neumann's test shows instability for all $r>0 .^{2}$

Presented to the Society, October 28, 1950; received by the editors July 21, 1950.

${ }^{1}$ Numbers in brackets refer to the references at the end of the paper.

${ }^{2}$ (Added in proof.) The above statements are based on $\$ \$ 8$ and 27 of [1]. In a recently published version of [1] in the Journal of Mathematics and Physics vol. 29 (1951) pp. 223-251 these sections have been revised and the convergence theorem of this paper is mentioned on page 238 as follows: "We remark without giving proof here, that $\Delta_{R}^{\prime}$ converges to $D$ as $\Delta x, \Delta t \rightarrow 0$, for all $r \equiv \Delta t / \Delta x^{2}$." With regard to this quotation reference is made to a forthcoming note by M. Hyman, Remarks on "A study of the numerical solution of partial differential equations." This note contains the passage, "The proof of convergence of $\Delta_{R}^{\prime}$ to $D$ mentioned on page 238 (lines 21, 22) is due to W. Leutert; it will appear in his paper, 'On the Convergence of Approximate Solutions of the Heat Equation to the Exact Solution', to be published in the Proceedings of the American Mathematical Society." 
In this paper the difference equation of Richardson will be considered and it will be shown that a proper choice of the initial condition to be satisfied by the approximation will imply convergence for all values of $r>0$. The fact that Richardson's solution does not converge for large values of $t$ is therefore not due to the choice of $r$ or the difference equation but to the choice of the initial condition and the procedure applied by Richardson to obtain a numerical solution.

Richardson's example is presently computed again by using a different numerical method based on the approximate solution given in this paper. It seems that similar statements can be proved for other difference equations which lead to the heat equation and for which von Neumann's test assures stability only for an $r$ less than a certain fixed constant.

The problem. Let a slab of homogeneous material bounded by the planes $x=0$ and $x=1$ have an initial temperature $u=f(x)$ varying only with the distance from the faces, and let its two faces be kept at temperature zero. The formula for the temperature $u(x, t)$ at every instant and at all points of the slab is to be determined.

It is no loss in generality to assume unit diffusivity.

The exact solution. The function $u(x, t)$ satisfies the differential equation

$$
u_{t}=u_{x x},
$$

the conditions at the end points of the bar

$$
u(0, t)=0 \quad \text { and } \quad u(1, t)=0, \quad t \geqq 0,
$$

and the initial condition

$$
u\left(x, 0^{+}\right)=f(x) \quad \text { for } 0<x<1 .
$$

The solution is well known and given by [3],

$$
\begin{aligned}
u(x, t) & =\sum_{n=1}^{\infty} a_{n} \sin n \pi x \exp \left(-n^{2} \pi^{2} t\right), \text { where } \\
a_{n} & =2 \int_{0}^{1} f\left(x^{\prime}\right) \sin n \pi x^{\prime} d x^{\prime} .
\end{aligned}
$$

It can be shown [3] that if $f(x)$ is sectionally continuous in the interval $(0,1)$ and has one-sided derivatives at all interior points of that interval, then $u(x, t)$ given by (1) is the unique solution of the problem. It will be called the exact solution. The conditions imposed 
on $f(x)$ are sufficient but not necessary.

An approximate solution. For the approximate solution to be considered the temperature will be denoted by $v(x, t)$. The difference equation

$$
\begin{aligned}
v(x, t+\Delta t)-v(x, & t-\Delta t) \\
& =2 r[v(x+\Delta x, t)+v(x-\Delta x, t)-2 v(x, t)], \\
r & =\frac{\Delta t}{(\Delta x)^{2}}
\end{aligned}
$$

is the same as the one used by Richardson [2]. The interval $(0,1)$ is divided into $M$ intervals of equal length $\Delta x$ so that for given $M$ and $r$

$$
\Delta x=\frac{1}{M}, \quad \Delta t=\frac{r}{M^{2}} .
$$

A formal solution of (2) satisfying the conditions

$$
v(0, t)=0 \text { and } v(1, t)=0
$$

is easily obtained by using the method of separation of variables

$$
v(x, t)=\sum_{n=1}^{\infty} b_{n} \sin n \pi x\left[\left(1+\alpha^{2}\right)^{1 / 2}+\alpha\right]^{-t M^{2} / r},
$$

where $\alpha=4 r \sin ^{2}(n \pi / 2 M), b_{n}$ arbitrary constants. Each term in (5) satisfies (2) and (4).

Since using one infinite series to approximate another infinite series does not simplify the problem too much, the following initial condition for $v(x, t)$ is chosen:

$$
b_{n}=0 \text { for } n>M, \quad b_{n}=a_{n} \text { for } 1 \leqq n \leqq M .
$$

This means that the function $u\left(x, 0^{+}\right)=f(x)$ is approximated by a trigonometric series in the sense of least squares.

The approximation has therefore the form

$$
\begin{aligned}
v(x, t) & =\sum_{n=1}^{M} a_{n} \sin n \pi x\left[\left(1+\alpha^{2}\right)^{1 / 2}+\alpha\right]^{-t M^{2} / r}, \\
a_{n} & =2 \int_{0}^{1} f\left(x^{\prime}\right) \sin n \pi x^{\prime} d x^{\prime}, \quad \alpha=4 r \sin ^{2} \frac{n \pi}{2 M} .
\end{aligned}
$$

$v(x, t)$ in (7) satisfies the difference equation (2) and the conditions (4) at the end points of the slab. In general

$$
v(x, 0) \not \equiv u(x, 0) \text {. }
$$


The convergence theorem in the next section applies also for values of $b_{n}$ slightly different from the $a_{n}$.

The convergence theorem.

Assumptions.

(1) $t \geqq 0, r>0,0 \leqq x \leqq 1, \alpha=4 r \sin ^{2}(n \pi / 2 M)$;

(2) to any $\epsilon>0$ there exists an $M_{0}(\epsilon)$ independent of $n$ such that for any $M>M_{0}$ and $1 \leqq n \leqq M$,

$$
\left|b_{n}(M)-a_{n}\right|<\epsilon / M
$$

TheOREM.

(10) $\lim _{M \rightarrow \infty} \sum_{n=1}^{M} b_{n} \sin n \pi x\left[\left(1+\alpha^{2}\right)^{1 / 2}+\alpha\right]^{-t M^{2} / r}=\sum_{n=1}^{\infty} a_{n} \sin n \pi x e^{-n^{2} \pi^{2} t}$, $x, t, r$ fixed.

Two lemmas. Let $h(z)=\left[\left(1+\alpha^{2}\right)^{1 / 2}+\alpha\right]^{-t M^{2} / r}-e^{-4 t M^{2} z^{2}}$ where $\alpha$ $=4 r \sin ^{2} z$, and $t \geqq 0, r>0$ are fixed constants while $M$ is a parameter.

LEMMA 1. $h(z) \geqq 0$ for any fixed $M$ and any $z$.

Proof. Since $h(z)=0$ for $t=0$, assume $t>0$. Consider

$$
g(z)=\sinh 4 r z^{2}-4 r \sin ^{2} z .
$$

Because $g(z)$ is even in $z, g(0)=0$, and

$$
g^{\prime}(z)=8 r z\left(\cosh 4 r z^{2}-\frac{\sin 2 z}{2 z}\right) \geqq 0 \quad \text { for } z \geqq 0,
$$

it follows that $g(z) \geqq 0$ or $\sinh 4 r z^{2} \geqq 4 r \sin ^{2} z$ which implies

$$
\left(1+\alpha^{2}\right)^{1 / 2}-\alpha=\frac{1}{\left(1+\alpha^{2}\right)^{1 / 2}+\alpha} \geqq e^{-4 r z^{2}}
$$

or

$$
\left[\left(1+\alpha^{2}\right)^{1 / 2}+\alpha\right]^{-t M^{2} / r} \geqq e^{-4 t M^{2} z^{2}}, \quad \text { q.e.d. }
$$

Lemma 2. For any $\epsilon>0$ there exists an $M_{1}(\epsilon, t)$ such that for any $M>M_{1}$ and $0 \leqq z \leqq \pi / 2$ we have $h(z)<\epsilon / M$.

Proof. Since $h(z)=0$ for $t=0$, assume $t>0$. Consider

$$
k(z)=4 r \sin ^{2} z+\sinh \beta \text { where } \beta=\frac{r}{t M^{2}} \ln \left(\frac{\epsilon}{M}+e^{-4 t M^{2} z^{2}}\right) .
$$

From 


$$
k^{\prime}(z)=8 r z\left[\frac{\sin 2 z}{2 z}-\frac{M \cosh \beta}{M+\epsilon e^{4 t M^{2} z^{2}}}\right]
$$

and $\sin 2 z / 2 z \geqq 1-2 z^{2} / 3$ for $0 \leqq z \leqq \pi / 2$ it follows that a sufficient condition that $k^{\prime}(z) \geqq 0$ is $2 z^{2} / 3+M \cosh \beta / M+\epsilon e^{4 \ell M^{2} z^{2}} \leqq 1$. For $0 \leqq z \leqq M^{-2 / 3}$ this inequality is certainly satisfied if

$$
\frac{2}{3} M^{-4 / 3}+\frac{M \cosh \left[r / t M^{2} \ln (M / \epsilon)\right]}{M+\epsilon} \leqq 1
$$

or

$$
\frac{2}{3} M^{-4 / 3}+\frac{1+r / t M^{2} \ln (M / \epsilon)}{1+\epsilon / M} \leqq 1
$$

for $\left(r / t M^{2}\right) \ln (M / \epsilon)<3 / 2$ because cosh $y \leqq 1+|y|$ for $|y|<3 / 2$.

Therefore

$$
\frac{2}{3} M^{-1 / 3}\left(1+\frac{\epsilon}{M}\right)+\frac{r}{t M} \ln \frac{M}{\epsilon} \leqq \epsilon .
$$

It is obvious that there exists an $M_{2}(\epsilon, t)$ such that, for any $M>M_{2}$, (15) is satisfied and we have $k^{\prime}(z) \geqq 0$ for $0 \leqq z \leqq M^{-2 / 3}$ and $M>M_{2}$. Since $k(0)=\sinh \left[r / t M^{2} \ln ((\epsilon / M)+1)\right]>0$ it follows that

$$
k(z)>0 \text { for } 0 \leqq z \leqq M^{-2 / 3} \text { and } M>M_{2} \text {. }
$$

Now assume $M^{-2 / 3} \leqq z \leqq \pi / 2$. Then

$$
k(z) \geqq 4 r \sin ^{2}\left(M^{-2 / 3}\right)-\sinh \gamma \quad \text { where } \quad \gamma=\frac{r}{t M_{1}^{2}} \ln \frac{M}{\epsilon}
$$

It is obvious that there exists an $M_{3}(\epsilon, t)$ such that for any $M>M_{3}$ the inequality

$$
M^{1 / 3}>\frac{\pi^{2}}{8 t M^{1 / 3}} \ln \frac{M}{\epsilon}
$$

is satisfied, which implies

$$
4 r \sin ^{2}\left(M^{-2 / 3}\right) \geqq \frac{16 r}{\pi^{2} M^{4 / 3}}>\frac{2 r}{t M^{2}} \ln \frac{M}{\epsilon} \geqq \sinh \left[\frac{r}{t M^{2}} \ln \frac{M}{\epsilon}\right]
$$

because

$$
\sin \left(M^{-2 / 3}\right) \geqq \frac{2}{\pi} M^{-2 / 3} \quad \text { for } \quad M^{2 / 3} \geqq \frac{\pi}{2}
$$


and

$$
\sinh \left[\frac{r}{t M^{2}} \ln \frac{M}{\epsilon}\right] \leqq \frac{2 r}{t M^{2}} \ln \frac{M}{\epsilon} \text { for } \frac{r}{t M^{2}} \ln \frac{M}{\epsilon}<1 .
$$

We further assume that $M_{3}$ has been chosen so large that the above two conditions are also satisfied for any $M>M_{3}$.

Therefore

$$
k(z)>0 \text { for } M^{-2 / 3} \leqq z \leqq \frac{\pi}{2} \text { and } M>M_{3}
$$

If we put $M_{1}=\max \left(M_{2}, M_{3}\right)$, then it follows from (16) and (18) that

$$
\alpha=4 r \sin ^{2} z>-\sinh \left[\frac{r}{t M^{2}} \ln \left(\frac{\epsilon}{M}+e^{-4 t M^{2} z^{2}}\right)\right],
$$

which implies

$$
2 \alpha\left[\frac{\epsilon}{M}+e^{-4 t M^{2} z^{2}}\right]^{r / 1 M^{2}}>1-\left[\frac{\epsilon}{M}+e^{-4 t M^{2} z^{2}}\right]^{2 r / t M}
$$

or

$$
\frac{\epsilon}{M}+e^{-4 t M^{2} z^{2}}>\left[\left(1+\alpha^{2}\right)^{1 / 2}+\alpha\right]^{-t M^{2} / r}
$$

We have proved $h(z)<\epsilon / M$ for $0 \leqq z \leqq \pi / 2$ and $M>M_{1}$, q.e.d.

Proof of the convergence theorem. Under the assumptions made on $f(x)$,

$$
\left|\sum_{n=1}^{\infty} a_{n} \sin n \pi x\right|=\frac{1}{2}|f(x+0)+f(x-0)|<P
$$

where $P$ is a constant independent of $x$.

If $z=n \pi / 2 M$ is put into Lemma 1 and Lemma 2, it follows immediately that for any $M>M_{1}$ and $1 \leqq n \leqq M$,

$$
\frac{\epsilon}{M}>\left[\left(1+\alpha^{2}\right)^{1 / 2}+\alpha\right]^{-t M^{2} / r}-e^{-n^{2} \pi^{2} t} \geqq 0, \quad \alpha=4 r \sin ^{2} \frac{n \pi}{2 M} .
$$

The convergence of the series used for $u(x, t)$ in (1) implies that there exists an $M_{4}(\epsilon, x, t)$ such that for any given $\epsilon>0$ and any $M>M_{4}$,

$$
\left|\sum_{n=1}^{\infty} a_{n} \sin n \pi x e^{-n^{2} \pi^{2} t}-\sum_{n=1}^{M} a_{n} \sin n \pi x e^{-n^{2} \pi^{2} t}\right|<\epsilon
$$


for fixed $x$ and fixed $t$. Now suppose that we chose

$$
M>\max \left(M_{0}, M_{1}, M_{4}\right) \text {. }
$$

Then for fixed $x$ and $t$

$$
\begin{aligned}
\mid \sum_{n=1}^{M} b_{n} & \sin n \pi x\left[\left(1+\alpha^{2}\right)^{1 / 2}+\alpha\right]^{-t M^{2} / r}-\sum_{n=1}^{\infty} a_{n} \sin n \pi x e^{-n^{2} \pi^{2} t} \mid \\
= & \mid \sum_{n=1}^{M}\left(b_{n}-a_{n}\right) \sin n \pi x e^{-n^{2} \pi^{2} t} \\
& +\sum_{n=1}^{M}\left(b_{n}-a_{n}+a_{n}\right) \sin n \pi x\left\{\left[\left(1+\alpha^{2}\right)^{1 / 2}+\alpha\right]^{-t M^{2} / r}-e^{-n^{2} \pi^{2} t}\right\} \\
& -\sum_{n=M+1}^{\infty} a_{n} \sin n \pi x e^{-n^{2} \pi^{2} t} \mid \\
\leqq & +\epsilon^{2}+\epsilon P+\epsilon \leqq \epsilon(P+3), \quad \text { q.e.d. }
\end{aligned}
$$

Additional results. Since in (15) and (17) $t$ appears only in the combination $t M^{2}$ or $t M^{1 / 3}$, the value of $M_{1}(\epsilon, t)$ is not increased by increasing $t$. Furthermore it can be shown [3] that under the assumptions made on $f(x)$ in (1) the series for $u(x, t)$ in (1) converges uniformly in $x$ and $t$ for $0 \leqq x \leqq 1$ and $0<t_{0} \leqq t$ where $t_{0}$ is a fixed positive constant. Therefore, in (22), $M_{4}=M_{4}\left(\epsilon, t_{0}\right)$ whenever $t \geqq t_{0}$.

Thus the limit in the convergence theorem is uniform in $x$ and $t$ for $0 \leqq x \leqq 1$ and $t_{0} \leqq t$, where $t_{0}$ is any positive constant.

\section{REFERENCES}

1. G. O'Brien, M. Hyman, and S. Kaplan, A study of the numerical solution of partial differential equations, Project NR 044-049*, Naval Ordnance Laboratory Memorandum 10433 (1949).

2. L. F. Richardson, The approximate arithmetical solution by finite differences of physical problems involving differential equations, Philos. Trans. Roy. Soc. London. Ser. A vol. 210 (1910) pp. 307-357.

3. R. V. Churchill, Fourier series and boundary value problems, New York, McGraw-Hill, 1941, chap. VI.

UNIVERSity OF MARYLAND 